\title{
Automated in situ line of sight calibration of ASDEX Upgrade bolometers
}

\author{
F. Penzel ${ }^{\mathrm{a}}$, H. Meister ${ }^{\mathrm{a}}$, M. Bernert ${ }^{\mathrm{a}}$, T. Sehmer ${ }^{\mathrm{a}}$, T. Trautmann ${ }^{\mathrm{a}}$, M. Kannamüller ${ }^{\mathrm{a}}$, J. Koll ${ }^{\mathrm{a}}$, A. W. Koch ${ }^{\mathrm{b}}$, and the \\ ASDEX Upgrade Team ${ }^{\mathrm{a}}$ \\ ${ }^{a}$ Max-Planck-Institute for Plasmaphysics, EURATOM Association, Garching, Germany \\ ${ }^{b}$ Institute for Measurement Systems and Sensor Technology, Technische Universität München, Germany
}

\begin{abstract}
The ITER Bolometer Robot Test Rig (IBOROB) is a robot-based diagnostic tool, which allows the measurement of the lines of sight (LOS) of the ITER bolometer prototypes. Up to now, it was only used as a LOS characterization device for the ITER collimator development. IBOROB was further developed and can now be operated in ASDEX Upgrade during a regular maintenance shutdown. At present, once a diagnostic like the bolometry is mounted inside the vessel, the actual LOS orientations are not measured, they are derived from CAD. The new procedure allows the fully automatic three-dimensional in situ measurement of bolometer LOS. The spatial distribution, the poloidal and toroidal alignment in the experiment coordinate system (CS), can be determined. The absolute accuracy, in reference to the tokamak CS, is provided by an additional calibration performed with a measurement arm by FARO Technologies Inc. Therefore, the amount of misalignment from the theoretical expectations can be quantified. In addition specific camera type dependencies such as internal camera reflections can be identified. Due to the high position accuracy of the robot, the LOS can be resolved with a spatial resolution of up to $0.1^{\circ}$. The method is explained in detail and results from two exemplary bolometer foil cameras obtained in a first set-up in ASDEX Upgrade are presented. The different steps and components needed to apply the measurements in the vessel are described with a focus on the constraints, e.g. geometrical, for an application of this method in a tokamak. Finally the consequences of the results are extrapolated to ITER and evaluated.
\end{abstract}

Keywords: ASDEX Upgrade, measurement by laser beam, bolometer, remote handling, LOS, robot

\section{Introduction}

Bolometry is a diagnostic which provides spatially resolved measurements of the radiated power in fusion experiments like ASDEX Upgrade. They are used as an indicator of the plasma purity and its confinement and provide fundamental data to perform transport studies. Main output is the determination of the local and global power balance. This diagnostic performs an integrated intensity measurement along its line of sight (LOS). Tomographic reconstruction algorithms can be applied on the measurement in order to retrieve the radiation distribution of a poloidal cross section. Especially for ITER, bolometers will be a key technology to control the quantity of the radiation in the divertor limiting the heat load on the divertor blanket modules [1] [2]. Therefore, the geometrical properties, the alignment of the LOS and their characteristics, are key parameters for the accuracy

Email address: florian.penzel@ipp.mpg.de (F. Penzel) of the diagnostic. Particularly in the divertor, the measurements of current day experiments are often poorly resolved in space and magnitude [3].

In ASDEX Upgrade the position of the bolometer camera in the vessel is measured with a coordinate measuring machine or at JET via photogrammetry [4]. However, only the orientation of the exterior camera housing can be determined, not the LOS itself. At present, the internal orientation of the LOS are then derived from CAD drawings and have to rely on the manufacturing accuracy. The alignment accuracy is probably within the range of a few degrees. But an exact lower boundary has not been proven yet and can only be estimated. As a consequence this introduces uncertainties in the results, in particular from the physics perspective. Another disadvantage is, that the angular étendu, which is used to absolutely calibrate the diagnostic for the measurement of the radiated power, usually is calculated from a simple geometric model [5]. Because of the complexity of e.g. multiple reflections, ray tracing 
calculation of the LOS have only been done on a simplified basis [6] [7]. An exact calculation of the camera is challenging due to the difficulty to model and quantify correctly all material properties, for instance to determine exactly, in the extremely wide spectral operating range of bolometers [8], the reflection coefficients.

These is one of the reasons that the results from bolometric power measurements in JET are not trusted to a higher accuracy than $\sim 10 \%$ [9]. Irregularities in the measurements and consequently in the tomographic reconstructions from ASDEX Upgrade are known but not fully understood. It is anticipated that some of the LOS are not aligned as expected or have imperfections in their characteristics [10]. Advanced numerical methods were developed in the past to improve the precision of the determination of the radiated power, but naturally this is limited by the quality of the input parameters [11]. In the end, upgrading the system, e.g. adding numerous cameras, is limited due to financial reasons and space restrictions. The amount of cameras and thereby the amount of cables should be minimized regarding the necessary amount of cables and vacuum vessel feedtroughs. These constraints are of particular interest for ITER as well.

In this paper a methodology to increase the measurement accuracy of the bolometers by directly measuring the alignment and characteristics of the LOS in the experiment is presented. The actual orientation of the LOS in the torus coordinate system (CS) will be determined at an angular measurement accuracy of $0.1^{\circ}$, independently of the inaccuracies of the camera assembly, installation procedure or other yet unknown errors. A precise knowledge of the LOS alignment will improve and facilitate the tomographic reconstruction. Thus, a more accurate prediction of the total radiated power is expected taking into account the actual orientation. However, internal vessel reflections of the plasma radiation on the first wall or the divertor tiles cannot be excluded from the measurement and will still affect the measurement accuracy of the diagnostic and create artifacts.

In the following paragraph the experimental setup will be described. In section 3 , results from two exemplary bolometer foil cameras are presented and the paper concludes with an outlook focused on the assessment of the results which influence ITER.

\section{Experimental set-up}

The measurements presented here were conducted during a regular vessel maintenance shutdown of ASDEX Upgrade. The measurement tool ITER Bolometer Robot Test Rig (IBOROB) was used for the

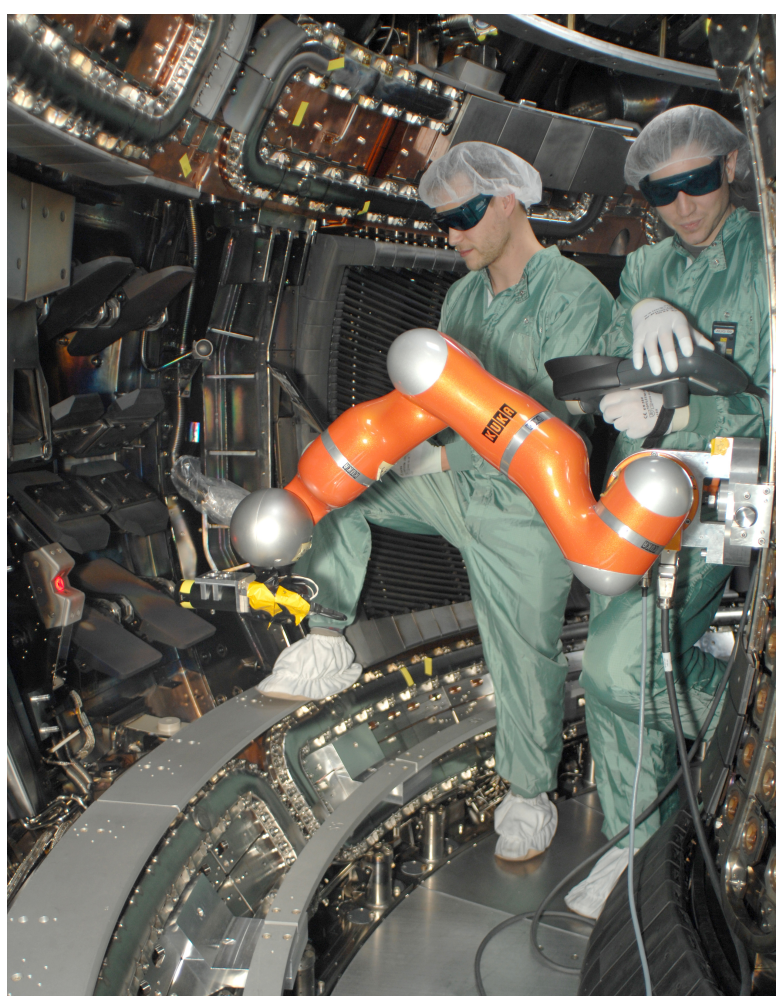

Figure 1: Picture of operating IBOROB in ASDEX Upgrade. Two scientists are inside the vessel. One person (right) is holding the main control of the robot and the other (on the left) is configuring the measurement parameters. The laser radiating on the FHC camera can be seen as a red circle on the left.

calibration measurements presented here. The tool was once developed for the measurement and assessment of the ITER bolometer diagnostic [7] and is based on work conducted at JET [12] [13]. Experience to analyse and evaluate the results were gained during the collimator development for ITER [14]. IBOROB was then completely automatized with National Instruments LabVIEW and the Imaging Lab Robotics Library for KUKA [15] and can be controlled completely through a GUI [16] in the automatic mode of the robot. The necessary programs can be remotely loaded and executed along with the definition of the measurement parameters.

In figure 1 the measurement system including the mounting device and two scientists operating the system in ASDEX Upgrade can be seen. The red circle on the left is the laser currently aiming on the bolometer camera named FHC. To conduct the measurements in situ, the robot was attached to a custom made mounting device which can be seen in figure 2. To optimally position IBOROB in the limited space between 

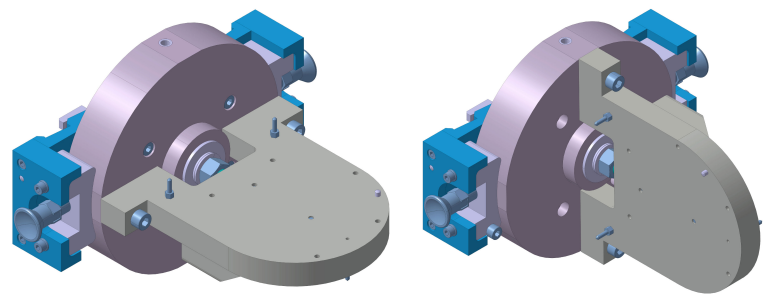

Figure 2: IBOROB mounting device for the inner heat shield of ASDEX Upgrade. One central rotating axis allows four different orientations of the measurement tool. Two positions are shown here (left:upward, right:turned right around $90^{\circ}$ )

major and minor radius of the torus for conducting the required movements, the device can be rotated in $90^{\circ}$ steps. The mounting device is manufactured from up to $15 \mathrm{~mm}$ thick stainless steel plates to withstand the leverage forces which can occur during fast accelerations such as an emergency stop of the robot. The total weight of the robot with payload amounts to $\sim 17 \mathrm{~kg}$.

In order to facilitate the measurement process and allow the comparison of LOS from different cameras without the need of re-calibrating the system, a fixed orientation of the mounting device was selected which is not changed during the whole measurement process. The mounting device rotated to the left was selected as the optimal position because of the possibility to access as many LOS as possible from three different cameras.

The principle of the measurement procedure is described in [7] and [14] in detail. For the measurements presented in this paper the LOS were measured with IBOROB moving in three- dimensional space. Along with the bolometer measurements the two dimensional transmission function in the toroidal and poloidal orientation can be calculated.

The ASDEX Upgrade foil bolometer system consists of six cameras using the pinhole construction design.

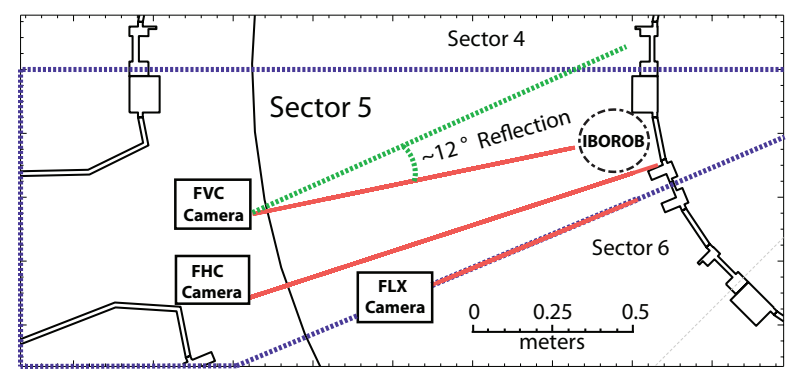

Figure 3: Toroidal distribution of ASDEX Upgrade bolometer foil cameras FVC, FLX and FHC - LOS are drawn as red lines. The location of IBOROB is indicated as a circle. The measured reflection is drawn as a green line.

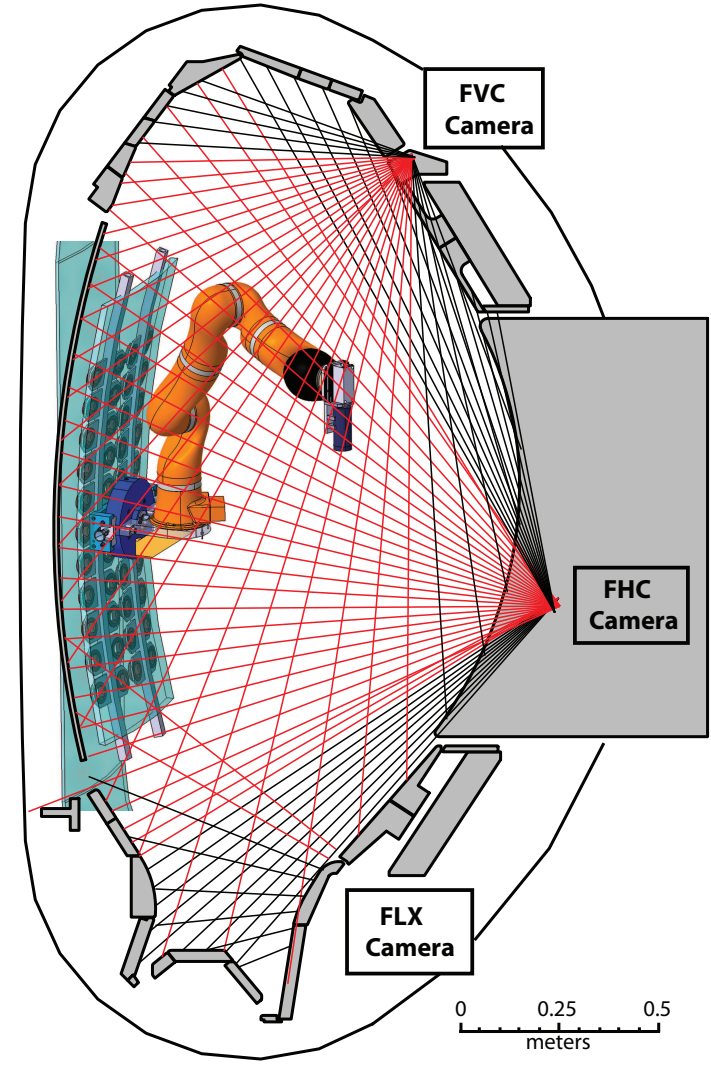

Figure 4: Poloidal cross section of sector 5 in ASDEX Upgrade. All LOS from the bolometer foil cameras FVC, FLX and FHC are plotted. The red lines indicate which LOS are accessible with IBOROB. The boxes indicate the approximate positions of the cameras in the vessel.

Two cameras located in the divertor are impossible to reach with the current setting and one camera mounted on the inner head shield was too close to the current mounting position in sector 5 . The scanning area would not have been sufficient. But three cameras have LOS which are accessible by IBOROB in this position. Two of these are discussed in this paper. Certain cameras (FHC/FVC) have multiple pinhole apertures. Due to the fact, that each pinhole contains a certain number of LOS, they have to be measured individually. In figure 3 the toroidal and in figure 4 the poloidal distribution of these bolometer camera LOS are shown. Those drawn in red could be measured. The position of IBOROB in relation to the vessel is indicated as well. The two cameras FHC and FVC were selected for the first measurements because they allowed the simplest access. They are both approximately radially in front of the robot. FHC is in the port area and the FVC near the upper passive stabilizing loop.

To determine the orientation of IBOROB and its in- 
ternal CS and to allow significant global statements from the results, the position of the system has to be absolutely calibrated. The exact orientation of the torus $\mathrm{CS}$, IBOROB and the positions of the pin-holes of the cameras have to be determined with high precision. To this aim the FaroArm ${ }^{\circledR}$ Platinum [17] is used. It is a portable coordinate measurement machine which allows in situ coordinate measurements over large volumes. In the current set-up a sufficient volumetric accuracy of $0.1 \mathrm{~mm}$ has been achieved. The measurement arm is integrated into the ASDEX Upgrade CS via measurement of multiple calibration points which are evenly distributed in the vessel. As a next step, the position of IBOROB and the cameras can be determined: The robot foot position is measured to determine the origin of the robot $\mathrm{CS}$. The orientation in relation to the vessel is then determined separately. It is done by conducting movements of the end effector along the $\mathrm{X}, \mathrm{Y}$ and $\mathrm{Z}$ axis. From these two measurements the robot CS within the torus CS can be constructed and calculated. The measurement distance between laser and bolometer aperture is a free parameter. Here it was optimized to maximize the poloidal or toroidal scanning range with the universal condition to not collide with any other vessel components. Finally, it is a trade-off between scanning many LOS and enabling a broad toroidal scanning range for the specific camera. At a measurement distance of $\approx 300 \mathrm{~mm}$ and after the calibration process an angular measurement accuracy of $0.1^{\circ}$ has been achieved.

An example of the scanning movement is shown in figure 5: the toroidal and poloidal orientation of the laser position in the bolometer CS versus time. The record is from a section of the movement during the measurement of the FHC camera (see chapter 3). From the start position, the robot makes one poloidal step followed by a complete toroidal scan. Then the robot moves back to the initial toroidal position. Subse-

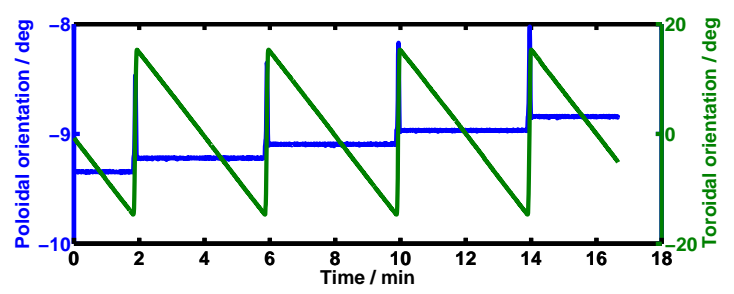

Figure 5: IBOROB motion during measurement procedure: Toroidal (green on the right) and poloidal (blue on the left) orientation in the robot CS versus time. After each toroidal scan of $\Delta \Phi=30^{\circ}$ a small poloidal step of $0.15^{\circ}$ is done. The spikes in the poloidal orientation are due to the PTP backwards movement. quently multiple toroidal slices of the LOS can be measured. During the re-positioning of the robot after each toroidal scan the poloidal alignment is shortly not maintained. The robot is limited in his orientation or degrees of freedom (DOF) only when it is necessary, here when measurement data is acquired. At other points in time the internal robot control algorithm is given the maximum possible freedom of optimally align the seven available axis.

Simultaneously after each alignment change $\left(0.15^{\circ}\right.$ steps for FHC and FVC measurement results) the bolometer data acquisition (DAQ) is triggered. The DAQ continuously acquires data, during the repositioning in toroidal direction as well, which results in the outlier data points of the poloidal axis plot of figure 5 (e.g. Time $=14 \mathrm{~min}$ or $10 \mathrm{~min}$ ) and is also the reason of the measurement noise which can be seen as scattered blue points in the results plot on figure 7. Applying a strict filter on the raw data, respectively deleting all data during re-positioning, these outliers can be filtered, but as not every data point contributes to the noise more data then necessary would be lost. Here, the data has not been altered in order not to affect the statements that can be derived from the measurements and with regard to optimizing the LabVIEW control program.

To obtain a measure of the deposited power, the DAQ needs to wait a constant idle time in the order of a few thermal time constants of the detector to reach the thermal equilibrium. Herein, one thermal time constant of the detector amounts to approximately $100 \mathrm{~ms}$. Then 500 samples of the bolometer bridge voltage are acquired and the average value is taken to realize a signal smoothing. Hence, the measurements are conducted in the steady state of the detector. Usually the incident power is calculated from the bolometer bridge voltage, its derivative over time and three material constants [18]. But for measurements where only relative statements are necessary, the bolometer bridge voltage can be used as a measure of the intensity of deposited power on the detector, which simplifies the DAQ.

A major requirement is to avoid singularities in the robot kinematic. Kinematic singularities of robot manipulators are configurations in which a change of DOF during the movement occurs. If a singularity is present in IBOROB's joint space, it can profoundly affect the performance, usually it results in a breakdown of the control algorithm which stops the robot automatically. In the environment of a VV like ASDEX Upgrade the robot already has a very limited work space. Moreover the robotic task conducted here restricts the orientation of the end effector additionally in two more DOF (poloidal and toroidal orientation of end effector) which 
makes it particularly problematic. Therefore, the robot was programmed using point to point (PTP) and not linear relative movements in the KUKA Robot Language KRL. The internal robot control algorithm can then optimize the path planning by freely determining all movements, apart from the current step in toroidal or poloidal orientation. This restricts the robot only in one DOF which results in being more fail-safe.

The downside of using PTP movements is that proper rectangular scanning areas cannot be defined exactly as can be seen in the measurement result plots in section 3. Furthermore, because of the constant signal acquisition and the fast toroidal backward PTP movement after each toroidal slice the measurement can not be associated to known coordinates and contributed to noise in the results. Only those signals acquired at steady positions of the robot give correct results. However, this error contributes to less then $1 \%$ of the acquired signals. For future measurements a filter will be integrated in the DAQ.

\section{Results and discussion}

Measurement results from two bolometer foil cameras, FHC and FVC, will be presented. The measured bolometer bridge voltage from the investigated detector channel is normalized to its maximum of the corresponding channel. The data is then plotted versus the poloidal (orientation in the vertical plane) and toroidal (orientation in the horizontal plane) angle, thus representing the normalized transmission function. All results presented here have been converted into the ASDEX Upgrade torus CS. The ASDEX Upgrade torus system is organized in 16 toroidal sectors each one spanning over $22.5^{\circ}$. IBOROB is positioned in the center of sector 5 which is at $\Phi=101.25^{\circ}$. The FAROArm determined the actual position of IBOROB to be at $\Phi=101.7^{\circ}$. All further required calculations for the coordinate transformations were done using the features of the FARO CAM $\mathrm{M}_{2}$ MEASURE software.

In figure 6 , a contour plot of the measurement results of the FHC bolometer channels 16 to 24 can be seen. This camera offers the opportunity to measure several channels within a small region. Each channel is tagged in the plot. Channel 18 had a broken detector, thus no measurement could be obtained. The measurement of channel 19 and 20 are strongly influenced by noise and thus the signals are only shown for relative values higher than $50 \%$ of the maximum to filter bitflip errors. The bolometer DAQ is usually used during the usual discharge times of $\sim 10 s$ of ASDEX Upgrade. Performing measurements with a duration of up to $24 \mathrm{~h}$

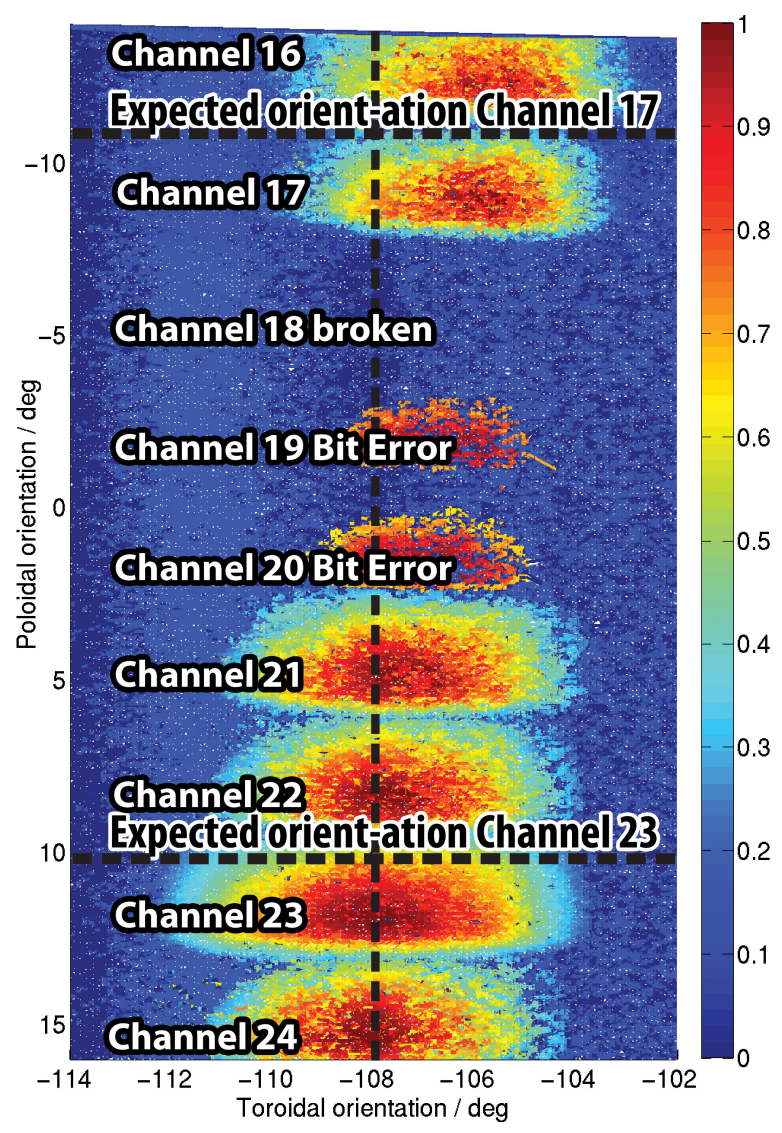

Figure 6: Contour plot of the calibration measurement of the FHC bolometer camera. Channels 16 to 24 are plotted versus the torus CS. Channel 18 is broken. The DAQ in Channel 19 and 20 had to many bit errors - only values above 50 percent are plotted. The expected orientations of Channel 17 and 23 are indicated as well.

reveled that a faulty analog to digital converter (ADC) from the DAQ electronics introduced these errors. On some channels (19 and 20) it had a strong impact, others e.g channel 23 remained nearly unaffected.

However, the channels 16/17/21/22/23/24 are shown completely. Due to the signal convolution of the rectangular aperture with the circular and Gaussian laser power distribution a rounded trapezoidal signal shape is expected and can be identified as well. The channels still show some difference in the geometrical properties but this might be due to the individual properties of the detectors. Some LOS maximums are dislocated from the center. Similar behavior could be observed during lab measurements in the past when the laser was shifted on purpose out of the center of the aperture. In the current experiment this shift resulted in a systematic error. The measurement shows also that the toroidal width is differing from Channel 17 to 23 . Unfortunately this 
could not be explained yet.

The dotted lines were added into the plot to indicate the expected orientation of Channel 17 and 23. They allow the comparison of the measurement with the theoretical expectations. In the toroidal orientation the FHC camera channels are expected at $\Phi=108^{\circ}$. This is very well satisfied for channel $22 / 23 / 24$. Due to a small inclination, presumably of the whole camera or more likely of single detector holders, channel 16/17/18 deviate slowly from the expected orientation. Channel 21-24 and channel 20-17 are independent detectors. Each detector contains 4 channels and is mounted next to each other in the camera housing, for those reason different alignments of the detectors influence only a selection of LOS which is probably the case for these channels. A horizontal tilt of the camera would only explain an equal misalignment of all channels. In the end it is only possible to measure the sum of all influencing parameters. The individual error sources cannot be definitely separated.

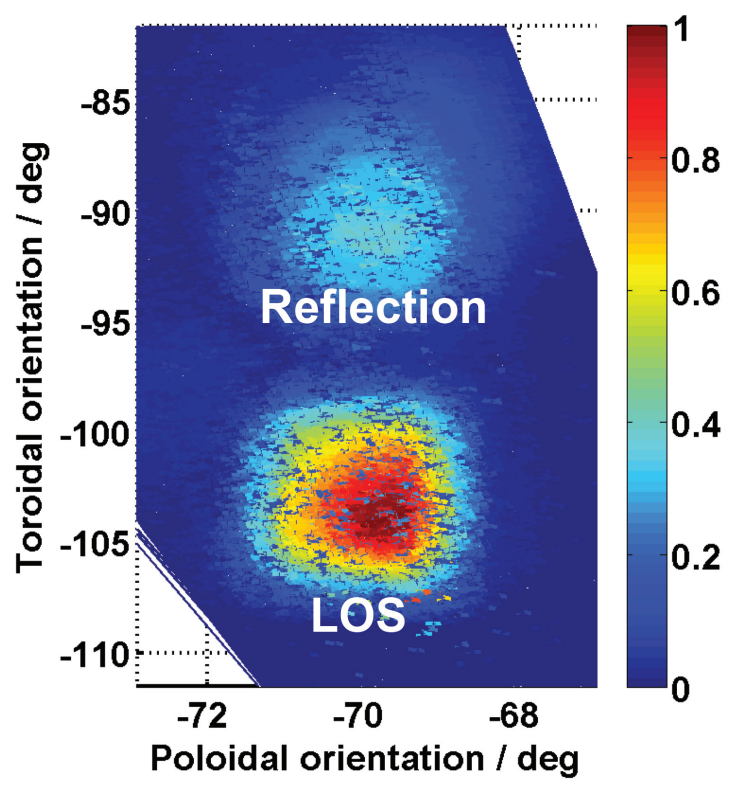

Figure 7: Contour plot of single channel of FVC Camera: Internal camera reflection can be identified. They are generated by the camera housing, which is made of reflective stainless steel plates.

In figure 7 the measurement result of a single LOS from the FVC camera is shown in a contour plot. The main objective of this measurement was to maximize the effective toroidal measurement range in order to detect signal artifacts such as reflections. The measurement does show a 'secondary', less intense signal response at a toroidal angular distance of $\sim 12^{\circ}$. This is created by a reflection of the incident light on the inner part of the camera housing, which is made of mirror-like stainless steel plates. Its direction is also indicated in the toroidal cross section in figure 3 . The reflection has an intensity of $30 \%$ compared to the maximum of the main signal and can be expected in the whole visible spectral range due to the similar reflection coefficients in this spectrum (638 nm wavelength of the laser). To make assumptions about its fraction in broader spectral ranges, the specific reflection coefficients of the material, the surface properties and the spectral power distribution have to be known. Each LOS with that characteristic will measure an additional radiated power. Other cameras used in ASDEX Upgrade are based on the same design and consequently suffer from reflections in poloidal orientation as well. Depending on the orientation of the respective LOS, the impact on the measured radiated power can be compensated with an adaptation of the calibration factors of the angular étendu and will be rather small due to the toroidal symmetry of the plasma. But in the case of LOS observing the divertor region with strong spatial variations of the plasma radiation density, the actual signal might be increased by a factor of 4 .

The noise, which can be seen in particular in figure 7 is due to the point to point relative movement during the measurement process and is not related to the bit-flip error. When the robot moves from one poloidal position to the next, there is a short time period where the laser is not aiming any more on the bolometer camera. Since the DAQ does not stop acquiring samples and the backward movement is much faster than during the normal measurement process the synchronization of position and bolometer signal gets lost. The signals are then assigned to the wrong position.

\section{Conclusions and outlook}

IBOROB was deployed for the first time in a tokamak environment and demonstrated its capabilities: Multiple LOS were measured automatically and directly in the vessel (in situ) independently from the geometric construction properties of the diagnostic with an angular uncertainty of $\sim 0.1^{\circ}$. During the last experimental campaign a few of the bolometer cameras have been upgraded due to the assumption of the reflective behavior of the internal camera housing. Not all cameras, only the ones which were easily accessible have been modified: Stainless steel plates with grooved surfaces were clamped on the inside wall of the camera housing with the aim to diffuse and scatter back radiation from unwanted angles of incidence. From the measurements of the unmodified cameras this assumption has been 
confirmed. The results show that previous accuracy assumptions considering the geometrical properties based on theoretical calculations should be considered with care.

One of the next steps at ASDEX Upgrade will be to analyse the channel étendu and compare the distances between adjacent channels. The complete two dimensional transmission function of the ASDEX Upgrade bolometers will be calculated for each camera head and then compared with the results. Currently, the calculation of tomographic pictures still requires experience, knowledge about tokamak physics, the experimental parameters and at times even a specific selection of LOS for proper reconstruction [10]. In the next campaign some of these constraints might be resolved due to this measurement tool.

Eventually it will be possible to identify further errors such as deviations from the expected trapezoidal form or to find a reason for the major deviations in the LOS width. It took a long time for the operators of the diagnostic to establish a connection between the unexpected measurement data acquired from certain LOS and the reflections in the camera housing. If IBOROB gets integrated in the regular maintenance process during each large vessel shutdown, a part of the diagnostic functionality could be checked in the future before experiment start up.

Another objective will be to detect occlusions of single LOS e.g. by plasma facing components in front of them. As the tiles in ASDEX Upgrade were already dismounted from the vessel during this measurement campaign such effects could not be observed, yet. Though it is planned to investigate this as soon as possible.

The first usage of IBOROB in ASDEX Upgrade revealed caveats: It took several days conducting the measurement presented here. Each camera, respectively aperture, had to be measured consecutively. Since the system must absolutely not collide with other diagnostics, wall elements or other experiments conducted in parallel inside the vessel, additional automatic movements like the re-positioning to a new start position between measurements of different cameras were avoided. After gaining experience operating the system in ASDEX Upgrade, the next step is to further automatize the measurement process allowing e.g. the consecutive measurement of multiple cameras without the need of manual human intervention.

It can be concluded, that during the development and construction phase of a new optical diagnostic with LOS characteristics the alignment issue during the installation should not be neglected. The results presented in this paper from the bolometer foil cameras FHC and
FVC arouse interest to conduct more measurements with the remaining bolometer cameras. The analysis of other diagnostics with similar optical designs might contribute to new insights in optical engineering of fusion diagnostics.

Deviations in the range of $2^{\circ}-3^{\circ}$, such as determined in the measurements presented here, are not a serious problem for medium-size tokamaks like ASDEX Upgrade. But if the vessel is increased in size by a factor of $\sim 4$, like for ITER, these deviations will cause severe problems. For example a poloidal misalignment of $3^{\circ}$ for a camera LOS with a length up to $7 \mathrm{~m}$ looking from the top into the divertor adds up to an error of $36 \mathrm{~cm}$. Misaligned LOS monitoring areas with strong gradients, such as the area around the X-point, will produce a significant blurring in the reconstructed radiation profile.

However, the major concern for the ITER bolometer LOS alignment is the following: The bolometer cameras, mounted on the inner structure of the vessel wall, will have to observe the plasma from behind large plasma facing components (PFC) with a thickness of $0.5 \mathrm{~m}$. To allow a correct measurement, the LOS will have to pass through these unaffected. The current plasma-facing components configuration [19] projects a nominal gap size between the blanket modules of $10-20 \mathrm{~mm}$ in order to respect nuclear shielding and remote handling requirements.

Current design considerations [20] propose nonstandard toroidal gap sizes of $30 \mathrm{~mm}$ at specific areas, e.g. for the gap between the divertor cassettes or between the PFC's to respect the LOS requirements. Defining the total width of the LOS at the PFC to be $28 \mathrm{~mm}$ and thus allowing for an alignment uncertainty at the gap of $1 \mathrm{~mm}$ this leads to a maximum total uncertainty in the orientation alignment of ITER in vessel components of $\arctan (1 \mathrm{~mm} / 500 \mathrm{~mm})=0.1^{\circ}$

An error in the range of the misalignment measured in ASDEX Upgrade will result in a ITER LOS with a channel étendu of merely half the expected value or in the worst case being completely obscured. If partly shadowed LOS remain unidentified the quality of the diagnostic system will be reduced. Even if quantities such as the total emitted power can be determined with a subset of the channels, for performing dependable tomographic reconstructions, a full set of reliable LOS is desirable.

Precision requirements for the ITER plasma control system [21] with contribution to advanced control or the accurate profile reconstruction for physics investigations will not be easily achievable. Therefore it should be considered to develop and integrate a similar system 
for ITER.

\section{Acknowledgments}

This work was supported by funds from the German Ministry for Education and Research under the Grant No. 03FUS0006. The authors wish to explicitly thank the student J. B. Calia for his assistance in data processing. The sole responsibility for the content presented lies with the authors.

\section{References}

[1] A. Huber, K. McCormick, P. Andrew, M. de Baar, P. Beaumont, S. Dalley, J. Fink, J. Fuchs, K. Fullard, W. Fundamenski, et al., Improved radiation measurements on JET - First results from an upgraded bolometer system, Journal of Nuclear Materials 363365 (2007) $365-370$.

[2] R. Neu, A. Kallenbach, M. Balden, V. Bobkov, J. Coenen, R. Drube, R. Dux, H. Greuner, A. Herrmann, J. Hobirk, et al., Overview on plasma operation with a full tungsten wall in ASDEX Upgrade, Journal of Nuclear Materials 438 (2013) 34 41.

[3] L. C. Ingesson, D. J. Wilson, Optimization of apertures and collimators for multi-channel plasma diagnostics, Review of Scientific Instruments 73 (8) (2002) 2890 - 2899.

[4] K. McCormick, A. Huber, C. Ingesson, F. Mast, J. Fink, W. Zeidner, A. Guigon, S. Sanders, New bolometry cameras for the JET enhanced performance phase, Fusion Engineering and Design 74 (2005) $679-683$.

[5] C. Fuchs, K. F. Mast, A. Herrmann, K. Lackner, ASDEX Upgrade Team, Twodimensional reconstruction of the radiation power density in ASDEX Upgrade, Europhysics Conference Abstracts, Proc. of the 21th EPS Conference on Controlled Fusion and Plasma Physics, Montpellier 1308 - 1311.

[6] L. C. Ingesson, P. J. Böcker, R. Reichle, M. Romanelli, P. Smeulders, Projection-space methods to take into account finite beam-width effects in two-dimensional tomography algorithms, Journal of the Optical Society of America 16 (1) (1999) $17-27$.

[7] H. Meister, F. Penzel, L. Giannone, M. Kannamüller, A. Kling, J. Koll, T. Trautmann, Development of an automated method for in situ measurement of the geometrical properties of the ITER bolometer diagnostic, Fusion Engineering and Design 86 (68) (2011) 1170 - 1173, Proceedings of the 26th Symposium of Fusion Technology (SOFT-26).

[8] H. Meister, M. Willmeroth, D. Zhang, A. Gottwald, M. Krumrey, F. Scholze, Broad-band efficiency calibration of ITER bolometer prototypes using $\mathrm{Pt}$ absorbers on $\mathrm{SiN}$ membranes, Review of Scientific Instruments 84 (12) (2013) 123501 - 7.

[9] L. C. Ingesson, Limitations to total radiated power determination in divertor tokamaks, in: JETP (99) 44, 1999.

[10] M. Bernert, Analysis of the H-mode densitylimit and fast edge phenomena with AXUV bolometry, Ph.D. thesis, LudwigMaximilians-Universität München (2013).

[11] L. C. Ingesson, Comparison of methods to determine the total radiated power in JET, in: JETR (99) 06, 1999.

[12] A. Huber, K. McCormick, P. Andrew, P. Beaumont, Upgraded bolometer system on JET for improved radiation measurements, Fusion Engineering and Design 8 (2007) 1327 - 1334.
[13] L. C. Ingesson, C. F. Maggi, R. Reichle, Characterization of geometrical detection-system properties for two-dimensional tomography, Review of Scientific Instruments 71 (3) (2000) 1370 $-1378$.

[14] F. Penzel, H. Meister, L. Giannone, M. Kannamüller, J. Koll, T. Trautmann, A. W. Koch, Assessment of line of sight characteristics of ITER bolometer prototype collimators, Fusion Engineering and Design 88 (6-8) (2013) 1267 - 1270, Proceedings of the 27th Symposium of Fusion Technology (SOFT-27).

[15] IMAGINGLAB Vision \& Robotics, Robotics Library for KUKA, Toolkit for Directly Commanding and Controlling KUKA Robotics from NI LabVIEW (2011).

[16] F. Penzel, H. Meister, L. Giannone, Der ITER Bolometer Roboter Prüfstand IBOROB: Automatisierte Sichtlinienvermessung mit NI LabVIEW, Virtuelle Instrumente in der Praxis 2013.

[17] Faroarm®Manual - Includes Quantum, Platinum, Fusion and ScanArm®models, FARO EUROPE GmbH \& Co. KG.

[18] L. Giannone, K. Mast, M. Schubert, Derivation of bolometer equations relevant to operation in fusion experiments, Review of Scientific Instruments 73 (9) (2002) 3205 - 3214.

[19] M. Merola, D. Loesser, A. Martin, P. Chappuis, et al., ITER plasma-facing components, Fusion Engineering and Design, Proceedings of the Ninth International Symposium on Fusion Nuclear Technology 85 (2010) 2312 - 2322.

[20] S. Kalvin, Optimisation of the ITER bolometer lines-of-sight and performance analysis, Final report to EFDA contract 031116, Association EURATOM-HAS, 2006.

[21] J. Snipes, Y. Gribov, A. Winter, Physics requirements for the ITER plasma control system, Fusion Engineering and Design 85 (3) (2010) $461-465$. 